\title{
Impact of COPD Exacerbation Frequency on Costs for a Managed Care Population
}

\author{
Anand A. Dalal, PhD, MBA; Jeetvan Patel, MS; Anna D'Souza, BPharm, PhD; Eileen Farrelly, MPH; \\ Saurabh Nagar, MS; and Manan Shah, PharmD, PhD
}

\begin{abstract}
BACKGROUND: There is scarce information on chronic obstructive pulmonary disease (COPD) outcomes and costs for patients with differing levels of COPD exacerbations.
\end{abstract}

OBJECTIVE: To examine COPD-related and all-cause health care resource use and costs in subsequent years for frequently and infrequently exacerbating COPD patients.

METHODS: Patients with a diagnosis of COPD (ICD-9-CM codes 491.xx, 492.xx, and 496.xx) were identified (1 hospitalization or 1 emergency department visit or at least 2 outpatient visits) using administrative claims data in 2007. Patients were classified in 2008 as frequent (at least 2 exacerbations/year), infrequent (1 exacerbation/year) and nonexacerbators. Outcomes were computed during a subsequent 2-year period (2009 and 2010). Average per person estimates and total sample-level estimates were calculated. A logistic regression model estimated the predictors of having 2 or more exacerbations per year during the follow-up period.

RESULTS: 61,750 COPD patients met the study criteria (mean age 67 years). Of these, $6 \%(n=3,852)$ were frequent exacerbators; $14 \%$ were infrequent exacerbators $(n=8,416)$; and $80 \%$ were nonexacerbators $(n=49,482)$. At baseline, average all-cause health care costs per patient for frequent exacerbators were highest followed by infrequent and nonexacerbators (\$12,837, $\$ 10,480$, and $\$ 7,756$, respectively). On average, $60 \%$ of frequent and $40 \%$ of infrequent exacerbators had at least 1 exacerbation per year in follow-up. Average annual per patient COPD-related costs for frequent exacerbators ( $\$ 3,565$ in 2009 and $\$ 3,528$ in 2010) were more than 3 times $(P<0.05)$ and infrequent exacerbators $(\$ 2,264$ in 2009 and $\$ 2,265$ in 2010) were more than 2 times $(P<0.05)$ higher compared with nonexacerbators (\$1,007 in 2009 and $\$ 1,027$ in 2010). On a total sample-level, infrequent exacerbators were similar if not more burdensome compared with frequent exacerbators in the proportion accounted by these cohorts for total COPD-related costs ( $23 \%$ vs. $18 \%$, respectively) and total number of COPD exacerbations per year ( $26 \%$ vs. $26 \%$ ). Compared with nonexacerbators, infrequent exacerbators were 3 times $(0 \mathrm{R}=2.8, P<0.001)$ significantly more likely to have 2 or more exacerbations per year in follow-up, and frequent exacerbators were 7 times $(0 R=6.76, P<0.001)$ significantly more likely to have 2 or more exacerbations per year in follow-up.

CONCLUSIONS: Infrequent exacerbators have an increased risk for future exacerbations compared with nonexacerbators and, on a total sample-level, incur greater costs compared with frequent exacerbators, demonstrating a significant economic burden.

J Manag Care Spec Pharm. 2015;21(7):575-83

Copyright $\odot 2015$, Academy of Managed Care Pharmacy. All rights reserved.

\section{What is already known about this subject}

It is well established that exacerbation frequency increases with chronic obstructive pulmonary disease (COPD) disease severity. Large cohort studies such as ECLIPSE have helped identify distinct exacerbator phenotypes in COPD patients, namely infrequent and frequent exacerbators.

\section{What this study adds}

This study further describes and quantifies the economic burden of the frequent and infrequent exacerbator phenotype in a realworld U.S. managed care setting.

Although frequent and infrequent exacerbators comprised 20\% of all COPD patients in this study, they accounted for $41 \%$ of all COPD-related costs and 55\% of exacerbations in a managed care population in subsequent years.

Management of infrequent exacerbators may be equally as important as the more severe frequent exacerbators, since, on a total sample-level, they incurred greater total costs compared with frequent exacerbators, demonstrating a significant economic burden.

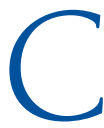

hronic obstructive pulmonary disease (COPD) is characterized by acute exacerbations, defined as events with worsening respiratory symptoms (e.g., sputum production, dyspnea, and cough) beyond the normal daily variation that a patient experiences, which leads to a change in medication. ${ }^{1}$ After an exacerbation, lung function does not completely recover, resulting in accelerated lung function decline, deteriorating quality of life, and increased risk of death. ${ }^{2-6}$ All of these factors contribute to the overall economic and societal burden of COPD exacerbations, particularly later in the disease process when rates of exacerbation are frequent. ${ }^{1}$

Although it is well established that exacerbation frequency increases as COPD severity increases, there is increasing evidence that a frequent exacerbator phenotype exists across all disease severity categories. ${ }^{7}$ The concept of a frequent exacerbator phenotype was first introduced in an observational study using data from the London COPD cohort, which found a cutoff of approximately 2 exacerbations per patient per year to more likely predict recurrent exacerbations. ${ }^{8}$ A recent pivotal observational study, Evaluation of COPD Longitudinally to Identify Predictive Surrogate Endpoints (ECLIPSE), further 
confirmed that a subset of COPD patients are likely to have more frequent exacerbations (2 or more exacerbations/year) regardless of disease severity. ${ }^{9}$ Based on the ECLIPSE study, the Global Initiative for Chronic Obstructive Lung Disease (GOLD) guidelines now consider exacerbations frequent when 2 or more events occur in a given year. ${ }^{1}$ In addition to the frequent exacerbator phenotype, ECLIPSE also evaluated infrequent exacerbators, defined as patients with only 1 or no exacerbations, and found that approximately 15\% of them had frequent exacerbations during follow-up. ${ }^{9}$

Data from ECLIPSE demonstrates that patients who experience multiple exacerbations within a year continue to be at high risk for future exacerbations. ${ }^{9}$ Previous studies have also shown that exacerbations are the primary drivers of cost within a manged care setting. Therefore, it is important to quantify how many patients with COPD experience multiple exacerbations within a year from a managed care perspective. Since the majority of patients with COPD do not have multiple exacerbations within a year, it is also essential to identify cost drivers among patients with COPD who experience infrequent or no exacerbations within a year.

The objective of the current study was to provide an estimate of the economic burden of the frequent and infrequent exacerbator phenotype in a real-world U.S. managed care setting, which is currently lacking in the literature. Specifically, the study evaluated the effect of patients characterized as frequent exacerbators ( 2 or more times per year), infrequent exacerbators (once per year), or nonexacerbators on health care resource use and costs, and subsequent exacerbation frequency, and additionally evaluated predictors of frequent exacerbations.

\section{Methods}

\section{Study Design and Sample Selection}

This retrospective cohort study used data from January 1 , 2007, to December 31, 2010 (study period), from the Truven Health MarketScan Commercial Claims and Encounters Database (Commercial) and the Medicare Supplemental and Coordination of Benefits Database (Medicare). Both databases include detailed cost, use, and outcomes data for health care services performed as inpatient and outpatient services and prescription drug claims, as well as information on patient enrollment. The Commercial database includes over 50 million individuals and represents the medical experience of insured employees and their dependents for active employees, early retirees, COBRA continuees, and their dependents insured by employer-sponsored plans (i.e., non-Medicare eligibles). The Medicare database contains claims data for approximately 3.1 million Medicare-eligible retirees with employer-sponsored Medicare Supplemental plans. Beneficiaries in the MarketScan Medicare database have drug coverage; therefore, drug data are available and provide additional valuable information.
Patients were identified as having COPD in 2007 based on the presence of 1 or more hospitalization or emergency department (ED) visit or 2 or more outpatient visits on different dates of service, with a diagnosis code for COPD in any field (International Classification of Diseases, Ninth Revision, Clinical Modification [ICD-9-CM] 491.xx [chronic bronchitis], 492.xx [emphysema], and 496.xx [chronic airway obstruction]). Next, these COPD patients were classified into the frequent, infrequent, and nonexacerbating phenotype (or cohorts in this case) using 2008 as the index year. The subsequent years (2009 and 2010) were then used to evaluate outcomes. Patients aged $\geq 40$ years with continuous eligibility for medical and pharmacy services from 2008 through 2010 were included in the study. Patients with specific comorbid respiratory conditions were excluded to avoid skewing the costs of the average COPD patient. Comorbidities were defined as a medical claim with a diagnosis or procedure code indicating the condition among patients with a confirmed diagnosis of COPD. These comorbidities were identified during the baseline and follow-up periods (list of conditions available from authors upon request).

\section{Study Cohorts}

Patients were categorized during the index year (2008) based on exacerbation frequency (frequent, infrequent, or nonexacerbator). A health care utilization-based definition for exacerbation was used in this study. ${ }^{10}$ Specifically, a COPD-related exacerbation was defined as the following: (a) hospitalization with a primary discharge diagnosis of COPD; (b) ED visit with a primary diagnosis of COPD; or (c) COPD-related physician visit with a primary diagnosis of COPD and receipt of a prescription for oral corticosteroids (OCS) or antibiotics within 5 days of the visit. ${ }^{11}$ Any of these encounters occurring within 14 days of each other were counted as 1 exacerbation, thus, requiring a gap of at least 14 days between encounters to count as separate exacerbations..$^{10}$ The frequent cohort included patients who had at least 2 exacerbations; the infrequent cohort included patients who had 1 exacerbation; and the nonexacerbator cohort included patients with no exacerbations during the index year (2008), ${ }^{1,9}$

\section{Study Outcomes}

Outcomes were summarized for individual follow-up years and the index year (where applicable to provide a point of reference) on a per-year basis for each exacerbator group. Outcomes included the proportion of patients with exacerbations and exacerbation rate (per patient per year) using the same health care use definition of exacerbation, health care resource use and costs, and other miscellaneous outcomes, such as 30-day and 60-day readmission and spirometry rates. Exacerbations were further described using a proxy-based measure of severity: severe (i.e., required hospitalizations) or moderate 
TABLE 1 Study Sample Description (Index Year 2008)

\begin{tabular}{|c|c|c|c|c|c|c|}
\hline \multirow[b]{2}{*}{ Characteristics } & \multicolumn{2}{|c|}{ Frequent Exacerbator } & \multicolumn{2}{|c|}{ Infrequent Exacerbator } & \multicolumn{2}{|c|}{ Nonexacerbator } \\
\hline & \multicolumn{2}{|c|}{$(\mathrm{N}=3,852)$} & \multicolumn{2}{|c|}{$(\mathrm{N}=8,416)$} & \multicolumn{2}{|c|}{$(\mathrm{N}=49,482)$} \\
\hline \multicolumn{7}{|l|}{ Demographic } \\
\hline Age, mean (SD) & 68.3 & $(10.5)$ & 68.3 & $(11.3)$ & 66.4 & $(12.2)$ \\
\hline \multicolumn{7}{|l|}{ Age group in years (n, \%) } \\
\hline $40-64$ & 1,248 & 32 & 3,000 & 36 & 21,865 & 44 \\
\hline $65-74$ & 1,384 & 36 & 2,581 & 31 & 12,693 & 26 \\
\hline$\geq 75$ & 1,220 & 32 & 2,835 & 34 & 14,924 & 30 \\
\hline Female (n, \%) & 2,191 & 57 & 4,591 & 55 & 25,630 & 52 \\
\hline \multicolumn{7}{|l|}{ U.S. geographical region (n, \%) } \\
\hline Northeast & 324 & 8 & 746 & 9 & 4,776 & 10 \\
\hline North Central & 1,534 & 40 & 3,344 & 40 & 17,897 & 36 \\
\hline South & 1,436 & 37 & 3,036 & 36 & 19,150 & 39 \\
\hline West & 555 & 14 & 1,281 & 15 & 7,625 & 15 \\
\hline Unknown region & 3 & 0 & 9 & 0 & 34 & 0 \\
\hline \multicolumn{7}{|l|}{ Physician specialty } \\
\hline Pulmonologist & 1,634 & 42 & 3,059 & 36 & 14,638 & 30 \\
\hline Internal medicine/family practice & 2,037 & 53 & 4,892 & 58 & 30,993 & 63 \\
\hline Other specialists & 126 & 3 & 326 & 4 & 2,786 & 6 \\
\hline Missing & 55 & 1 & 139 & 2 & 1,065 & 2 \\
\hline \multicolumn{7}{|l|}{ Overall comorbidity burden } \\
\hline CCI, mean (SD) & 2.2 & $(1.4)$ & 2.1 & $(1.4)$ & 1.6 & $(1.5)$ \\
\hline Asthma (n, \%) & 1,066 & 28 & 1,807 & 21 & 6,855 & 14 \\
\hline URTI (n, \%) & 896 & 23 & 1,730 & 21 & 8,325 & 17 \\
\hline LRTI $(n, \%)$ & 1,819 & 47 & 2,946 & 35 & 8,702 & 18 \\
\hline CVD (n, \%) & 2,307 & 60 & 4,919 & 58 & 26,954 & 54 \\
\hline Depression (n, \%) & 451 & 12 & 870 & 10 & 4,362 & 9 \\
\hline Number of Rx, mean (SD) & 17.8 & $(7.6)$ & 14.5 & $(7.3)$ & 10.0 & $(7.3)$ \\
\hline Number of unique Dx, mean (SD) & 16.3 & $(8.5)$ & 14.7 & $(8.2)$ & 11.8 & $(7.8)$ \\
\hline \multicolumn{7}{|l|}{ COPD severity } \\
\hline SABA canister use (n, \%) & 2,417 & 63 & 4,089 & 49 & 12,506 & 25 \\
\hline Number of SABA canisters, mean (SD) & 5.4 & $(7.4)$ & 3.4 & $(6.0)$ & 1.6 & $(4.3)$ \\
\hline OCS prescription use $(n, \%)$ & 3,287 & 85 & 5,137 & 61 & 10,013 & 20 \\
\hline Number of OCS prescriptions, mean (SD) & 3.3 & $(3.2)$ & 1.4 & $(2.1)$ & 0.4 & $(1.3)$ \\
\hline Use of home oxygen therapy (n, \%) & 1,564 & 41 & 2,405 & 29 & 8,299 & 17 \\
\hline ICU stay for COPD (n, \%) & 144 & 4 & 140 & 2 & 0 & 0 \\
\hline
\end{tabular}

(i.e., required an $\mathrm{ED}$ or physician visit with receipt of OCS or antibiotic within 5 days of the physician visit). All-cause and COPD-related costs and resource use were evaluated. Medical claims with a primary diagnosis of COPD and claims for COPD-specific drugs were used to compute COPD-related costs. Costs were estimated using the costs paid to the provider by the employer health plan and Medicare for the service after applicable discounts and any deductibles, coinsurance, and copayment tied to the existing ICD-9-CM codes on medical claims. All costs were standardized to the last year of available data (2010) using the medical component of the Consumer Price Index to ensure a common level of reference for costs measured in the study period. Medical resource use for all sites of care-hospitalizations, ED visits, physician visits, and out- patient visits-were evaluated, as well as use of COPD-related drugs, both maintenance and reliever. The 30- and 60-day all-cause readmission rate was calculated as the proportion of patients having a hospitalization with any diagnosis within 30 days (and 60 days) of the first hospitalization with a discharge diagnosis for COPD during the follow-up.

\section{Analysis}

A descriptive analysis was performed using means and standard deviations (medians as appropriate) and proportions for categorical variables to summarize the outcomes for the 3 types of exacerbators during the index and follow-up years. A cost estimate for the entire analytical sample was estimated. The total cost estimate for the sample was obtained by 

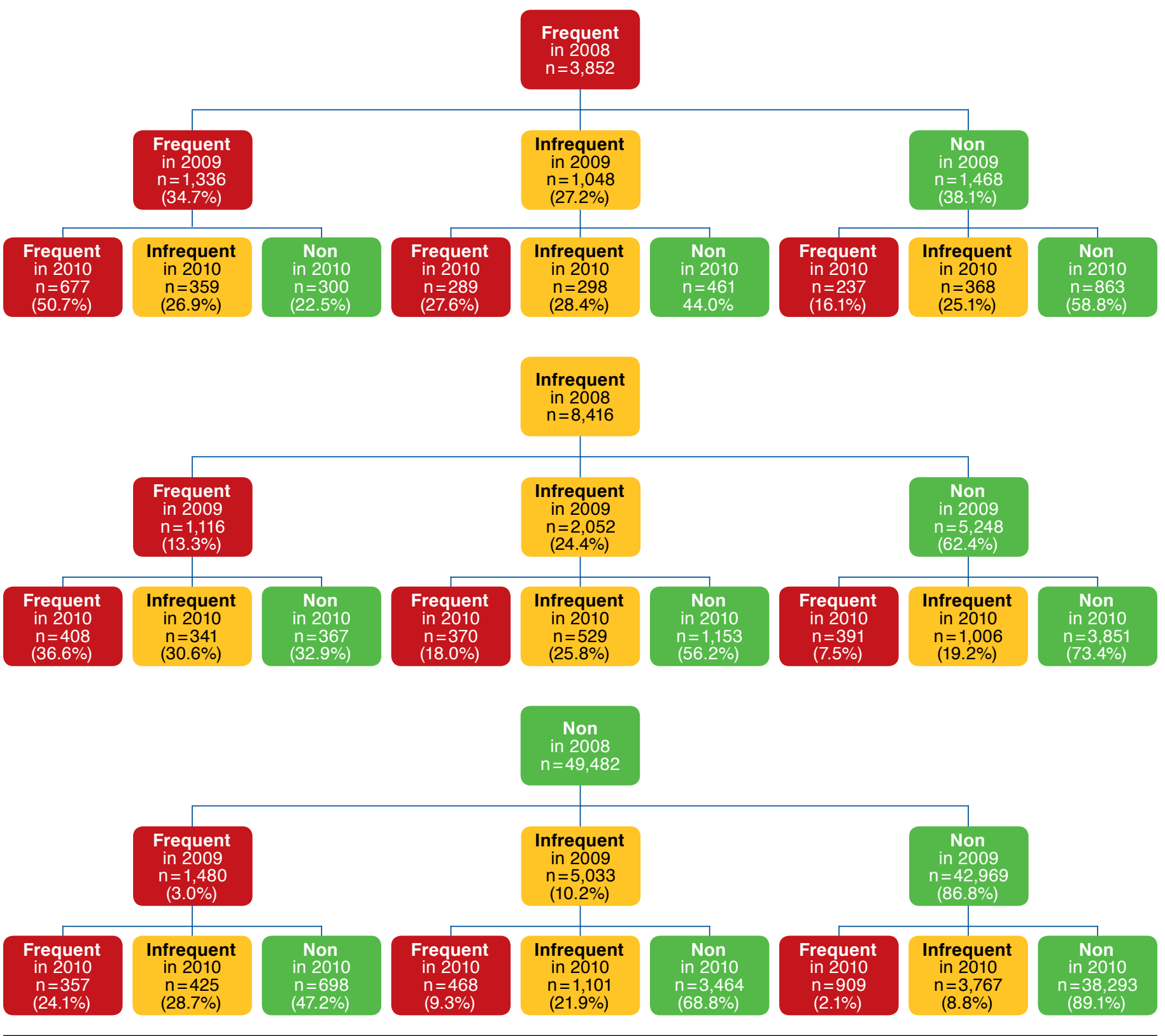

multiplying the average cost per patient per year and number of exacerbations per patient per year by the total number in each exacerbator cohort. The study used a logistic regression to evaluate the predictors of having frequent exacerbations. The outcome of interest was 2 or more exacerbations per year. This outcome was measured over a 2-year follow-up period (2009 and 2010). The key predictor of interest was the type of exacerbator (frequent, infrequent, and nonexacerbator). The nonex- acerbator cohort was used as the reference cohort. Other model covariates measured during the index year (2008) included presence of a severe or moderate exacerbation, demographic characteristics, overall comorbid burden, and proxies of COPD severity. All statistical tests were conducted using SAS version 9.1.3 (SAS Institute, Cary, NC), testing a 2-sided hypothesis at a significance level of 0.05 . 


\section{FIGURE 2 All-Cause and COPD-Related Costs Per Year by Exacerbator Type}

\section{A. All-Cause Costs}

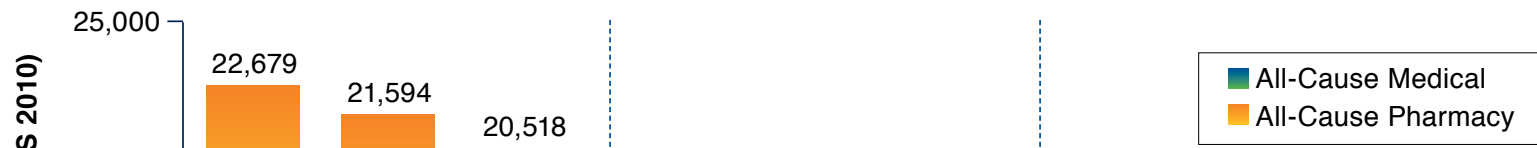

\section{B. COPD-Related Costs}

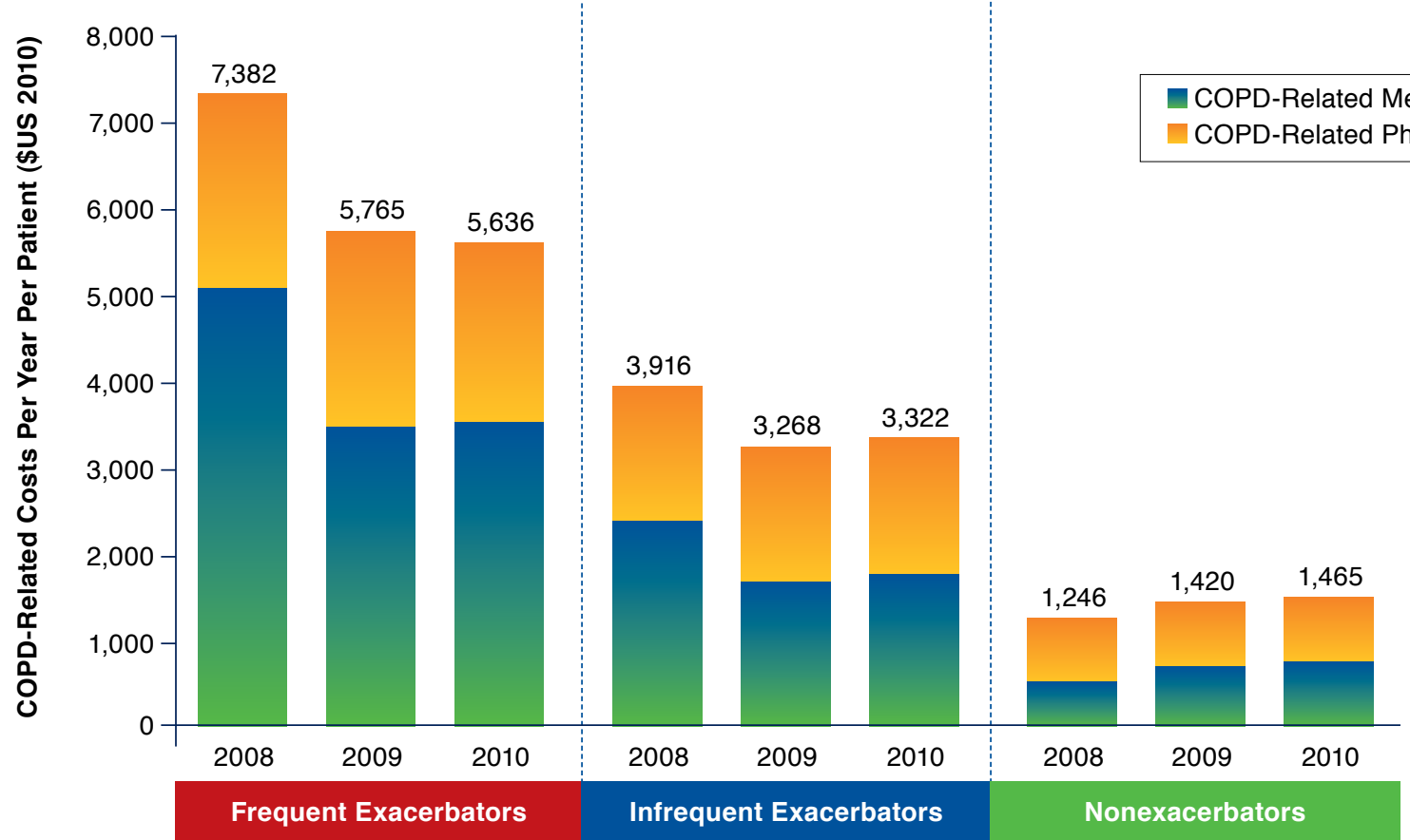

\section{Results}

A total of $145,741(66,147$ from the Commercial dataset and 79,594 with Medicare) patients with a diagnosis for COPD in 2007 were identified from the data source; 61,750 patients met all the study criteria and were included in the analysis. The majority of the study population was classified as nonexacerbators $(n=49,482 ; 80 \%) ; 6 \%(n=3,852)$ and $14 \%(n=8,416)$ of patients were frequent and infrequent exacerbators, 

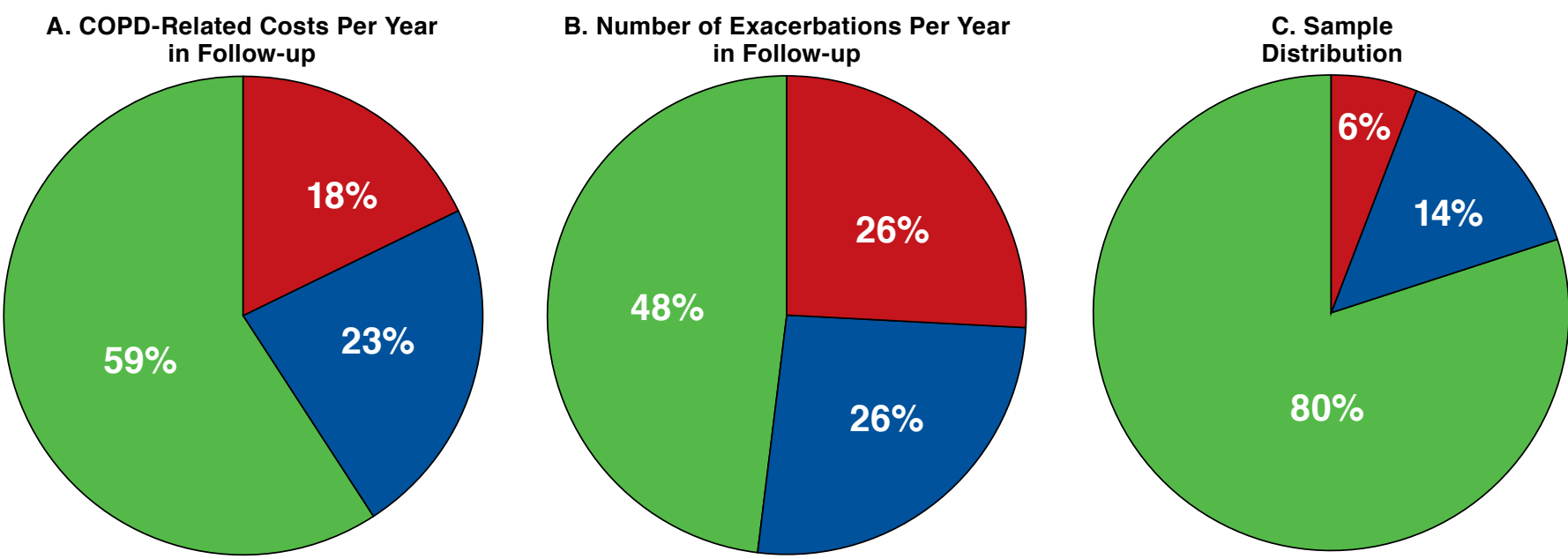

Frequent

Infrequent

Nonexacerbator

COPD $=$ chronic obstructive pulmonary disease

respectively. The average age of the study sample was 67 years with an equal representation of males and females (see Table 1). Frequent and infrequent exacerbators were similar in age and gender in contrast to nonexacerbators, who were relatively younger and more likely to be male. Frequent exacerbators had the highest proportion of patients with pulmonologist visits, followed by infrequent and then nonexacerbators. Frequent and infrequent exacerbators were similar on overall comorbidity burden metrics and had more comorbidity than nonexacerbators. At least half of frequent and infrequent exacerbators were using short-acting beta-agonists (SABA) compared with $25 \%$ of nonexacerbators.

\section{Exacerbations}

Over 2 years of follow-up, frequent exacerbators were more likely to experience at least 1 exacerbation per year over the follow-up period (78\%), compared with infrequent (54\%) and nonexacerbators (23\%; Figure 1). A distinct and increased risk of future exacerbations was noted among exacerbator types. In particular, risk of 2 or more exacerbations in 2009 was higher for frequent exacerbators, followed by infrequent and nonexacerbators (Figure 1: $35 \%$ vs. $13 \%$ vs. $3 \%$ ). A similar trend was noted when evaluating the risk in 2010 among those with frequent, infrequent, and no exacerbations in 2009 (37\% vs. $14 \%$ vs. $3 \%)$.

Overall, there seemed to be relative stability for the frequent exacerbator and nonexacerbator phenotypes, with $51 \%$ of those with frequent exacerbations in 2008 and 2009 also having frequent exacerbation in 2010 and $89 \%$ of those without any exacerbations in 2008 and 2009 having none in 2010.
However, only $25 \%$ of those with infrequent exacerbations in 2008 and 2009 continued to have infrequent exacerbations in 2010, indicating movement to other phenotypes. Specifically, infrequent exacerbators are more likely to continue to have 1 or more exacerbation than none (54\% vs. $46 \%$ ).

Appendix A (available in the online article) shows the exacerbation rate and prevalence per year during the follow-up period and index year (for a reference point). During the index year, frequent exacerbators experienced 2 or more exacerbations by definition (average 2.63). However, even during each of the follow-up years, frequent exacerbators continued to experience more than 1 exacerbation per year. Infrequent exacerbators also continued to experience approximately 0.6 exacerbations per year for each of the follow-up years compared with approximately 0.2 per year for nonexacerbators. The probability of a severe or moderate exacerbation increased with frequency of exacerbations (Appendix A). Notably, the probability doubled with the proportion of frequent exacerbators being almost twice that of infrequent exacerbators, which in turn was more than twice that of nonexacerbators experiencing either a moderate (2009: 57\% vs. $34 \%$ vs. $12 \%$ ) or severe exacerbation (2009: $15 \%$ vs. $7 \%$ vs. $2 \%$ ).

\section{All-Cause and COPD-Related Costs}

Figure 2 summarizes the average per person per year allcause and COPD-related costs. As expected, frequent exacerbators had the highest costs followed by infrequent and nonexacerbators for all-cause and COPD-related costs. Across all groups, the data show the costs to be constant across the follow-up years. On average, frequent exacerbators incurred approximately $\$ 7,400$ in COPD-related costs, accounting for 
a third of their all-cause costs. In contrast, COPD-related costs accounted for less than a quarter of all-cause costs of infrequent exacerbators and $10 \%$ for nonexacerbators. For all exacerbator types, medical costs accounted for the majority of all-cause costs (approximately $72 \%-75 \%$ of total). However, the proportion of COPD-related medical costs accounted for by total COPD-related costs differed by exacerbator type (60\%$70 \%, 50 \%-60 \%$, and $40 \%-50 \%$ for frequent, infrequent, and nonexacerbators, respectively) and remained constant during follow-up.

Figure 3 provides the total sample-level proportional distribution of costs and number of exacerbations. Frequent and infrequent exacerbators comprise $6 \%$ and $14 \%$ of all COPD patients, respectively, but account for a similar proportion of total COPD-related costs (23\% and $18 \%$, respectively) and total exacerbations (28\% and 26\%, respectively).

\section{Other Outcomes}

Treatment pattern analyses show that about 30\% of patients with a history of exacerbation were not identified as taking COPD maintenance medication. Maintenance medication use was the highest among patients with frequent exacerbations, followed by infrequent and nonexacerbators, respectively. This possibly may be due to exacerbation status in the prior year (2007) during which they were identified or perhaps because their disease severity level as noted using the $\mathrm{FEV}_{1} / \mathrm{FVC}$ ratio necessitated use of maintenance drugs. Across all exacerbator types, combination inhaled corticosteroids (ICS) plus long-acting beta agonist (LABA) drugs were most commonly used, followed by tiotropium, ipratropium, ICS, LABA, and xanthenes.

All-cause readmission rates were constant across the years (2008 to 2010). In the index year (2008), the frequent exacerbators had higher readmission rates: $4.7 \%$ compared with $1.4 \%$ for infrequent exacerbators and $0 \%$ for nonexacerbators. However, in the follow-up years, 30-day readmission rates for infrequent exacerbators were only slightly lower than frequent exacerbators. In 2009 , rates were $4.7 \%$ and $4.4 \%$ for frequent and infrequent exacerbators, respectively; in 2010, rates were $4.8 \%$ and $3.3 \%$, respectively. Frequent exacerbators were more likely to have lung function tested with spirometry compared with infrequent exacerbators. During the index year (2008), $42 \%$ and $32 \%$ of frequent and infrequent exacerbators, for example, used spirometry. In 2010, 31\% and $26 \%$ of patients, respectively, used spirometry. Across all types of exacerbators, however, the rates were less than half.

\section{Predictors of 2 or More Exacerbations in Follow-up Period}

Patients classified as frequent exacerbators during the index year (2008) were 7 times more likely than nonexacerbators to have 2 or more exacerbations per year in the follow-up period (odds ratio $[\mathrm{OR}]=6.60 ; 95 \%$ confidence interval $[\mathrm{CI}]=5.14$ 8.48; $P<0.001)$. Infrequent exacerbators were 3 times more likely than nonexacerbators to experience 2 or more exacerbations per year in follow-up $(\mathrm{OR}=2.68 ; 95 \% \mathrm{CI}=2.18$ 3.304; $P<0.001$ ). Other significant predictors (Appendix B, available in online article) included the following: aged $\geq 65$ years $(P<0.001)$, care by a pulmonologist $(P<0.001)$, upper respiratory tract infection $(P=0.002)$, lower respiratory tract infection $(P<0.001)$, depression $(P=0.028)$, SABA canister use $(P<0.001)$, OCS prescription use $(P<0.001)$, and home oxygen use $(P<0.001)$.

\section{Discussion}

The current study represents the first study, to the authors' knowledge, to evaluate the economic burden of frequent and infrequent exacerbators in a managed care setting. Although frequent and infrequent exacerbators comprise 20\% of all COPD patients, they continue to account for $41 \%$ of all COPDrelated costs and 55\% of exacerbations in a managed care population in subsequent years. It is important to point out that the current study used a relatively stringent definition of exacerbation, requiring a COPD-related visit in conjunction with use of OCS or antibiotics. Thus, it is likely that patients classified as nonexacerbators had exacerbations based solely on the use of OCS or antibiotics without a concomitant COPD-related visit. This difference in definition has the effect of underestimating the total proportion of exacerbators in our COPD population as seen by comparing our sample to that of ECLIPSE: higher proportion of nonexacerbators (80\% vs. approximately $46 \%$ ) and lower proportion of frequent (6\% vs. $29 \%$ ) and infrequent exacerbators (14\% vs. $25 \%)^{9}$

The overall study findings, however, were similar to the ECLIPSE study, albeit underestimated, which was likely because of differences in the definition of exacerbation. In particular, similar to ECLIPSE, the phenotype for frequent exacerbators was shown to be stable over a 3-year period, with $51 \%$ of those with frequent exacerbations in the first 2 years continuing to have frequent exacerbations in year 3 (compared with $71 \%$ in ECLIPSE). Unlike ECLIPSE, we differentiated infrequent exacerbators from nonexacerbators. Nonexacerbators were similar to frequent exacerbators in terms of the stability of exacerbation frequency, with $89 \%$ of those without exacerbation in the first 2 years continuing to have no exacerbations in year 3. However, our study found that more than half of infrequent exacerbators were likely to continue to have at least 1 exacerbation per year, with 13\% having 2 or more exacerbations per year. The difference in the exacerbation rate per year also indicates that infrequent exacerbators continue to demonstrate increased risk compared with nonexacerbators (0.6 vs. 0.2/year). Thus, in the opinion of the authors of this study, the infrequent exacerbator category of patients should not go unnoticed and may benefit greatly from effective management programs from providers, payers, and employers. 
Our study, like others, showed that a history of exacerbations predicted frequency of exacerbations. ${ }^{1,9}$ Other predictors reported in the literature, particularly for patients with infrequent exacerbations who switched to frequent exacerbations over a year's time, include worse baseline postbronchodilator-forced expiratory volume in 1 second $\left(\mathrm{FEV}_{1}\right), \mathrm{FEV}_{1} \%$ predicted, and anxiety. ${ }^{12}$ Predictors of frequent exacerbations noted in our study further contribute to areas where patient management and education may be useful.

\section{Limitations}

Our study results must be interpreted within the limitations of the study design and data source. Only exacerbations that resulted in a health care encounter were identified from the data source; consequently, mild exacerbations (mainly defined as presence of symptoms not requiring medical intervention) that may affect the day-to-day functioning of patients were not evaluated. Only patients with continuous eligibility were included; thus, patients who died were not evaluated. Therefore, the costs may be underestimated for patients with exacerbations who have a higher likelihood of mortality. Other limitations stem from using claims data that are primarily collected for reimbursement for health services and not for research purposes; therefore, issues of coding (either miscoding, upcoding, or undercoding) may have occurred. Additionally, coding for disease conditions may be due to ruling out diagnoses rather than actually coding for presence of the condition. Nevertheless, claims data afford opportunities for assessing large populations in real-world settings. Because of the data source, the generalizability of the results is limited. Specifically, the study population only included employer-sponsored commercially insured patients and Medicare patients with supplemental insurance. As such, study findings are not representative of other patient populations such as Medicaid and Medicare patients without supplemental insurance.

\section{Conclusions}

Results of our observational study demonstrate that patients with frequent and infrequent exacerbations comprise a relatively small portion of the overall COPD population, yet account for almost half of COPD-related costs. Therefore, patients with exacerbations, regardless of frequency, represent an important area of focus for health care providers, payers, and employers. Since COPD exacerbations impose a substantial economic and societal burden in the United States, these exacerbations need to be effectively assessed and managed, notably in patients with frequent exacerbations and, perhaps, equally important for patients with infrequent exacerbations.

\section{Authors}

ANAND A. DALAL, PhD, MBA, is Director, US Health Outcomes, GlaxoSmithKline, Research Triangle Park, North Carolina; JEETVAN PATEL, MS, is Senior Manager, Health Economics and Outcomes Research, Amgen, Thousand Oaks, California; and SAURABH NAGAR, MS, is Health Outcomes Scientist, RTI Health Solutions, Research Triangle Park, North Carolina. ANNA D'SOUZA, BPharm, PhD, is Associate Director, Applied Data Analytics, and EILEEN FARRELLY, MPH, is Associate Director, Applied Data Analytics, Xcenda, Palm Harbor, Florida. MANAN SHAH, PharmD, PhD, is Director, Health Economics and Outcomes Research, Bristol-Myers Squibb, New Brunswick, New Jersey.

AUTHOR CORRESPONDENCE: Anand A. Dalal, PhD, MBA, GlaxoSmithKline, 5 Moore Dr., B.3130, Durham, NC 27709.

E-mail: anand.a.dalal @gsk.com.

\section{DISCLOSURES}

Funding for this study (GHO-11-3324) was provided by GlaxoSmithKline.

Dalal is an employee of GlaxoSmithKline and owns company stock. Patel and Nagar were employed with GlaxoSmithKline at the time of this study. D'Souza and Farrelly are employees of Xcenda and received research funding from GlaxoSmithKline. Shah was employed with Xcenda at the time of this study.

Study concept and design were contributed by Dalal, D'Souza, Patel, Farrelly, and Shah. Data were collected by Farrelly, Dalal, D'Souza, Patel, and Shah and interpreted by Dalal, Patel, D'Souza, Farrelly, and Shah. The manuscript was written by D'Souza, Dalal, Patel, Farrelly, and Shah and revised by Dalal, Patel, D'Souza, Farrelly, and Shah.

\section{REFERENCES}

1. Global Initiative for Chronic Obstructive Lung Disease. Global strategy for the diagnosis, management, and prevention of chronic obstructive pulmonary disease. Updated 2014. Available at: http://www.goldcopd.org/uploads/ users/files/GOLD_Report2014_Feb07.pdf. Accessed June 9, 2015.

2. Donaldson GC, Seemungal TA, Bhowmik A, Wedzicha JA. Relationship between exacerbation frequency and lung function decline in chronic obstructive pulmonary disease. Thorax. 2002;57(10):847-52.

3. Seemungal TA, Donaldson GC, Paul EA, Bestall JC, Jeffries DJ, Wedzicha JA. Effect of exacerbations on quality of life in patients with chronic obstructive pulmonary disease. Am J Respir Crit Care Med. 1998;157(5 Pt 1):1418-22.

4. Miravitlles M, Ferrer M, Pont A, et al. Effect of exacerbations on quality of life in patients with chronic obstructive pulmonary disease: a 2 year follow up study. Thorax. 2004;59(5):387-95.

5. Soler-Cataluña JJ, Martinez-Garcia MA, Román-Sánchez PR, Salcedo E, Navarro M, Ochando R. Severe acute exacerbations and mortality in patients with chronic obstructive pulmonary disease. Thorax. 2005;60(11):925-31.

6. Donaldson GC, Wedzicha JA. COPD exacerbations: 1: epidemiology. Thorax. 2006;61(2):164-68.

7. Wedzicha JA, Donaldson GC. Exacerbations of chronic obstructive pulmonary disease. Respir Care. 2003;48(12):1204-13. 
8. Hurst JR, Donaldson GC, Quint JK, Goldring JJ, Baghai-Ravary R, Wedzicha JA. Temporal clustering of exacerbations in chronic obstructive pulmonary disease. Am J Respir Crit Care Med. 2009;179(5):369-74.

9. Hurst JR, Vestbo J, Anzueto A, Locantore N, et al. Susceptibility to exacerbation in chronic obstructive pulmonary disease. N Engl J Med. 2010;363(12):1128-38.

10. Burge S, Wedzicha JA. COPD exacerbations: definitions and classification. Eur Respir J Suppl. 2003;41:46s-53s.
11. Lee TA, Wilke C, Joo M, et al. Outcomes associated with tiotropium use in chronic obstructive pulmonary disease patients. Effective Health Care Research Report No. 15. (Prepared by Chicago-Area DEcIDE Center Under Contract No. HSA29020050038I TO4). Rockville, MD: Agency for Healthcare Research and Quality. December 2009. Available at: http://www.effectivehealthcare.ahrq.gov/index.cfm/search-for-guides-reviews-and-reports/?pageactio $\mathrm{n}=$ displayproduct\&productID=364\&returnpage $=$. Accessed June 1, 2015.

12. Donaldson GC, Müllerova H, Locantore N, et al. Factors associated with change in exacerbation frequency in COPD. Respiratory Res. 2013;14:79. 
APPENDIX A Prevalence and Rate of Exacerbations Per Year by Exacerbator Type and Severity

\begin{tabular}{|c|c|c|c|c|c|c|}
\hline \multirow[b]{2}{*}{ Exacerbator Type ${ }^{a}$} & \multicolumn{2}{|c|}{ Index Year (2008) } & \multicolumn{2}{|c|}{ Follow-up Period (Year 1, 2009) } & \multicolumn{2}{|c|}{ Follow-up Period (Year 2, 2010) } \\
\hline & Mean (SD) & $\begin{array}{c}\text { Percentage of } \\
\text { Exacerbator } \\
\text { Cohort Sample }\end{array}$ & Mean (SD) & $\begin{array}{l}\text { Percentage of } \\
\text { Exacerbator } \\
\text { Cohort Sample }\end{array}$ & Mean (SD) & $\begin{array}{l}\text { Percentage of } \\
\text { Exacerbator } \\
\text { Cohort Sample }\end{array}$ \\
\hline \multicolumn{7}{|l|}{ Frequent $(\mathrm{n}=3,852)$} \\
\hline All & $(1.1)$ & $100.0^{\mathrm{b}}$ & $(1.6)$ & 61.9 & $1.24 \quad(1.6)$ & 57.8 \\
\hline Severe & $(0.6)$ & 26.4 & $(0.5)$ & 14.5 & $0.19 \quad(0.5)$ & 15.1 \\
\hline Moderate & $2.29 \quad(1.1)$ & 97.8 & $(1.4)$ & 57.3 & $1.05 \quad(1.4)$ & 53.0 \\
\hline ED & $(0.8)$ & 28.7 & $0.20 \quad(0.6)$ & 14.2 & $0.21 \quad(0.6)$ & 14.6 \\
\hline Phy+Rx & $(1.2)$ & 87.8 & $0.95 \quad(1.3)$ & 50.2 & $0.84 \quad(1.2)$ & 45.5 \\
\hline \multicolumn{7}{|l|}{ Infrequent $(n=8,416)$} \\
\hline All & $1.00 \quad(0.0)$ & $100.0^{\mathrm{b}}$ & $0.59 \quad(1.0)$ & 37.6 & $0.59 \quad(1.0)$ & 36.2 \\
\hline Severe & $0.13 \quad(0.3)$ & 13.2 & $0.08 \quad(0.3)$ & 6.9 & $0.09 \quad(0.3)$ & 7.8 \\
\hline Moderate & $0.87 \quad(0.3)$ & 86.9 & $0.51 \quad(0.9)$ & 34.3 & $0.50 \quad(0.9)$ & 32.3 \\
\hline ED & $0.16 \quad(0.4)$ & 16.1 & $0.09 \quad(0.3)$ & 7.6 & $0.10 \quad(0.4)$ & 8.1 \\
\hline Phy+Rx & $(0.5)$ & 70.7 & $(0.8)$ & 29.1 & $0.40 \quad(0.8)$ & 26.9 \\
\hline \multicolumn{7}{|c|}{ Nonexacerbator $(n=49,482)$} \\
\hline All & N/A & N/A & $(0.5)$ & 13.2 & $0.19 \quad(0.6)$ & 14.2 \\
\hline Severe & N/A & N/A & $0.02 \quad(0.2)$ & 2.3 & $0.03 \quad(0.2)$ & 2.6 \\
\hline Moderate & N/A & N/A & $0.15 \quad(0.5)$ & 11.7 & $0.17 \quad(0.5)$ & 12.6 \\
\hline ED & N/A & N/A & $0.03 \quad(0.2)$ & 2.7 & $0.04 \quad(0.2)$ & 3.4 \\
\hline Phy+Rx & N/A & N/A & $0.12 \quad(0.4)$ & 9.5 & $0.13 \quad(0.4)$ & 10.0 \\
\hline
\end{tabular}




\section{APPENDIX B Predictors of 2 or More Exacerbations Per Year in Follow-up}

Characteristics

Odds Ratio

$95 \%$ CI

$P$ Value

Exacerbation type in 2008 (reference: nonexacerbator)

\begin{tabular}{l|l}
\hline Frequent & 6.603 \\
\hline Infrequent & 2.68 \\
\hline Presence of severe exacerbation in 2008 (reference: no) & 1.080 \\
\hline Presence of moderate exacerbation in 2008 (reference: no) & 1.072
\end{tabular}

\begin{tabular}{l|l}
6.603 & $(5.143-8.477)$ \\
\hline
\end{tabular}

\begin{tabular}{l|l}
6.684 & $(2.180-3.304)$ \\
\hline
\end{tabular}

1.080

$(0.925-1.260)$

Presence of moderate exacerbation in 2008 (reference: no)

1.072

$(0.870-1.322)$

$<0.0001$

Demographic

Age group in years (reference: $40-64$ years)

\begin{tabular}{l|l}
\hline $65-74$ & \\
\hline$\geq 75$ & \\
\hline Male (reference: female) &
\end{tabular}

\begin{tabular}{l|l}
1.410 & \\
1.181 & \\
1.042 &
\end{tabular}

\begin{tabular}{l|l}
$(1.314-1.514)$ & $<0.0001$
\end{tabular}

\begin{tabular}{l|r}
$(1.097-1.270)$ & $<0.0001$ \\
\hline
\end{tabular}

U.S. geographical region (reference: South)

\begin{tabular}{l|l}
\hline Northeast & 0.95 \\
\hline North central & 1.003 \\
\hline West & 0.95
\end{tabular}

Physician specialty (reference: internal medicine/family practice)

Pulmonologists

Other specialists

\begin{tabular}{|c|c|}
\hline & 55 \\
\hline & 03 \\
\hline
\end{tabular}

(0.983-1.104)

0.1670

Overall comorbidity burden

Charlson Comorbidity Index

Asthma (reference: no asthma)

URTI (reference: no URTI)

LRTI (reference: no LRTI)

CVD (reference: no CVD)

Depression (reference: no depression)

1.151

(0.858-1.063)

(0.940-1.071)

(0.876-1.045)

0.3327

0.5126

\section{COPD severity}

SABA canister use (referemce: no use)

OCS prescription use (reference: no use)

Use of home oxygen therapy (reference: no use)

\begin{tabular}{l|l}
1.1517 & $(1.085-1.222)$ \\
0.917 & $(0.795-1.058)$
\end{tabular}

(0.795-1.058)

\begin{tabular}{|l|l}
\hline 0.996 & \\
\hline 0.999 & \\
\hline 1.121 & \\
\hline 1.327 & \\
\hline 0.866 & \\
\hline 1.113 & \\
\hline
\end{tabular}

(0.976-1.017)

$(0.930-1.074)$

$(1.043-1.205)$

$(1.246-1.413)$

(0.815-0.921)

(1.011-1.224)

0.3980

0.9216

0.3239

$C C I=$ Charlson Comorbidity Index; $C I=$ confidence interval; $C O P D=$ chronic obstructive pulmonary disease; $C V D=$ cardiovascular disease; $L R T I=$ lower respiratory tract infection; OCS = oral corticosteriods; $S D=$ standard deviation; $U R T I=$ upper respiratory tract infection. 